\title{
Mesenchymal Stem Cell Soluble Mediators and Cystic Fibrosis
}

Morgan T Sutton ${ }^{1-3,6-8}$, David Fletcher ${ }^{1}$, Nicole Episalla ${ }^{1,4}$, Lauren Auster ${ }^{1,4}$, Michael Folz ${ }^{6}$, Varun Roy ${ }^{1,3}$, Rolf van Heeckeren ${ }^{1}$, Donald $\mathrm{P}$ Lennon ${ }^{4,5}$, Arnold I Caplan ${ }^{4,5}$ and Tracey L Bonfield ${ }^{1,2,3,5 *}$

${ }^{1}$ Department of Pediatrics, Case Western Reserve University, USA

${ }^{2}$ National Center of Regenerative Medicine, Case Western Reserve University, USA

${ }^{3}$ School of Medicine, Case Western Reserve University, USA

${ }^{4}$ Department of Biology Case Western Reserve University, USA

${ }^{5}$ Skeletal Research Center, Case Western Reserve University, USA

${ }^{6}$ School of Engineering, Case Western Reserve University, Cleveland Ohio, USA

${ }^{7}$ Hathaway Brown School, Shaker Heights, Ohio, USA

${ }^{8}$ Department of Pediatrics, Rainbow Babies and Children's Hospital, Cleveland Ohio, USA

\begin{abstract}
Human Mesenchymal stem cells (hMSCs) secrete products (supernatants) that are anti-inflammatory and antimicrobial. We have previously shown that hMSCs decrease inflammation and Pseudomonas aeruginosa infection in the in vivo murine model of Cystic Fibrosis (CF). CF is a genetic disease in which pulmonary infection and inflammation becomes the major cause of morbidity and mortality. Our studies focus on determining how hMSCs contribute to improved outcomes in the CF mouse model centering on how the hMSCs impact the inflammatory response to pathogenic organisms. We hypothesize that hMSCs secrete products that are anti-inflammatory in scenarios of chronic pulmonary infections using the murine model of infection and inflammation with a specific interest in Pseudomonas aeruginosa (gram negative). Further, our studies will identify whether the hMSCs are impacting this inflammatory response through the regulation of peroxisome proliferator activator receptor gamma (PPARY) which aides in decreasing inflammation.
\end{abstract}

Keywords: Mesenchymal stem cells; Chemokines; Cytokines; Antiinflammatory cytokines; Anti-inflammation; Chemotaxis; PPAR $\gamma$; Cystic fibrosis

\section{Introduction}

Mesenchymal Stem Cells (hMSCs) have been shown to be antiinflammatory and anti-microbial in vitro as well as in vivo using the murine model of CF lung infection and inflammation [1-3]. Cystic Fibrosis (CF) is a genetically inherited fatal disease in which the Cystic Fibrosis Transmembrane Conductance Regulator (CFTR) gene is mutated resulting in defective protein causing patients to have failure to thrive, lung congestion and infection with a variety of bacteria [4]. Defective CFTR results in impeded mucocilliary clearance, which is associated with increased susceptibility to bacterial infection requiring treatment with antibiotics as well as mucus thinning reagents [5]. Pseudomonas aeruginosa is the most prevalent bacteria in CF infections, but is not the only bacteria involved in creating serious infection and damage in the CF lung. Organisms such as Streptococcus pnuemoniae and Staphylococcus aureus create a complex pulmonary niche and microbiome [6,7]. In the end, despite new developments in small molecular correctors and CFTR expression enhancers, the ensuing inflammatory response, pulmonary failure continue to be a serious issue leading to significant morbidity and eventual mortality in $\mathrm{CF}$ [8]. The immune response in CF individuals has been shown to be excessive, and ultimately contributing to lung damage. In healthy lungs, bacterial infiltration results in acute neutrophil influx and eradication of bacteria followed by inflammation resolution. However, in CF patients, the neutrophil influx is excessive with increased release of neutrophils containing Neutrophil Elastase (NE), which is extremely damaging to the lung milieu. NE induces an increased gland secretion, and an increase in bacterial binding to the airway surface [9]. Increased levels of neutrophils also lead to an influx in oxygen radical production which induces chemokine productin including interleukin 8 (IL-8) which perpetuates further recruitment of neutrophils. hMSCs secrete products that are anti-inflammatory, anti-microbial, angiogenic, chemotactic, anti-apoptotic, and anti-scarring [10,11]. hMSCs have been shown to have therapeutic potential in redirecting the excessive $\mathrm{CF}$ and inflammatory based pathophysiology $[12,13]$. In these studies we studied hMSC efficacy in the infection/inflammation using the murine model of cystic fibrosis (CF)14. Wild Type (WT) and cystic fibrosis transmembrane Conductance Regulator (Cftr) Deficient Mice (CF) mice were chronically infected with Pseudomonas aeruginosa (PA) and followed for several days for clinical score, survival and weight loss kinetics. At euthanasia, Bronchoalveolar Lavage (BAL) showed that hMSCs shifted the pulmonary differential away from neutrophils towards macrophages which was statistically significant for CF animals since they traditionally have an augmented neutrophilic response. In the studies reported here, we have begun to pursue the mechanisms associated with the anti-inflammatory function of hMSCs in the Cftr deficient murine models. We report that the anti-inflammatory potency of the hMSCs can affect cell recruitment and aide in attenuating the robust response inherent in CF pathophysiology. Our data also suggest that the mechanism of anti-inflammatory action is through regulating the inflammatory transcription regulator PPAR $\gamma$ as well as the production of chemokines ultimately impacting the phenotype of the inflammatory response. We further provide data to suggest that deficient CFTR function alters hMSC's activity implicating the need for allogeneic sources of hMSCs for clinical trials. The novelty of the results

*Corresponding authors: Tracey L Bonfield, Ph.D. D(ABMLI), Associate Professor Department of Pediatrics Case Western Reserve University, Biomedical Research Building \#822, 10900 Euclid Avenue, Cleveland Ohio 44106-4948, USA, Tel: 216368-4558; Fax: 216-368-4223; E-mail: Tracey.Bonfield@case.edu

Received August 27, 2017; Accepted September 18, 2017; Published September 22, 2017

Citation: Sutton MT, Fletcher D, Episalla N, Auster L, Folz M, et al. (2017) Mesenchymal Stem Cell Soluble Mediators and Cystic Fibrosis. J Stem Cell Res Ther 7: 400. doi: 10.4172/2157-7633.1000400

Copyright: @ 2017 Sutton MT, et al. This is an open-access article distributed under the terms of the Creative Commons Attribution License, which permits unrestricted use, distribution, and reproduction in any medium, provided the original author and source are credited. 
in this manuscript demonstrate the unique niche which defines the function of hMSCs and their powerful therapeutic potential for diseases associated with chronic inflammation, in the context of unrelenting infection. Further we have begun to define the mechanistic impact of the hMSCs in the context of inflammation and how it might be impacted in scenarios of deficient CFTR activity.

\section{Methods}

\section{Human cell preparations}

hMSCs: hMSC preparations were obtained from the National Center for Regenerative Medicine, Case Comprehensive Cancer Center, and SCC Cellular Therapy Laboratory. The hMSCs were isolated and grown following the standard operating procedures associated with hMSCs in clinical trials $[15,16]$. hMSCs were expanded and grown in hMSC condition media treated with $10 \%$ fetal calf serum. hMSCs were taken from first or second passage.

PBMCs: Peripheral Blood Mononuclear Cells (PBMCs) were isolated from healthy control individuals as previously described using Institutional IRB approval [17]. We experimented with the protocol to optimize the yield of monocytes in anticipation of obtaining limited blood volumes from CF patients. The isolated PBMCs were either cultured with control media (RPMI 1641) or hMSC preparations (1:1). Control conditions of these cultures were left unstimulated and experimental conditions were stimulated with Lipopolysaccharide (LPS) at $10 \mu \mathrm{g} / \mathrm{ml}$. There was a 24 -hour incubation period at $37^{\circ} \mathrm{C}$.

Animal studies: All Animal Studies were done with approval by Case Western Reserve IACUC committee and approval of application 2011-0146. Cystic Fibrosis transmembrane receptor deficient animals (Cftrtm1Kth, CF) and congenic background controls (C57BL/6J, WT) were anesthetized and inoculated with agarose beads embedded with 106 Pseudomonas Aeruginosa (PA-M5715) or Staphylococcus Aureus (ATCC\# SA 29523).

Animals were followed with daily weights and clinical scores out to 10 days. Animals were euthanized and evaluated for lung inflammation by bronchoalveolar lavage (BAL) for cytokines, chemokines, cellular recruitment and differentials as described previously $[3,18]$.

Gene expression: hMSCs that were used both in the in vivo murine $\mathrm{CF}$ model and the in vitro bactericidal and anti-inflammatory assays were processed for messenger ribonucleic acids (mRNA) followed by synthesis of complementary deoxyribonucleic acid (cDNA) for chemokine gene expression by real-time-PCR [19]. hMSCs were grown in the presence and absence of CFTR inhibitor (I-172, $1 \mu \mathrm{g} / \mathrm{ml}$ ) to mimic CF hMSCs 3. Both baseline and CFTR inhibited hMSCs were stimulated with and without lipopolysaccharide (LPS, $10 \mu \mathrm{g} / \mathrm{ml}$ ) to mimic exposure to gram negative bacteria. The quality of mRNA and synthesized cDNA was assessed using Nanodrop spectrophotometry technology (optimal threshold $260-280 \mathrm{~nm}$ ) as well as validated using peptidyl prolyl isomerase A (PPIA) as the human ubiquitous housekeeping gene. We evaluated the expression of interleukin-8 (IL-8), interleukin 6 (IL-6), chemokine ligand 2(CCL-2), chemokine ligand 20 (CCL20) and peroxisome proliferator activated receptor gamma (PPAR $\gamma)$ as a comparison of PPIA ubiquitous gene expression. In the animal studies, gene expression was compared to glyceraldehyde 3-phosphate dehydrogenase (GAPDH). All data is expressed as deltaCT response using PPIA (hMSCs) or GAPDH (RAW 642.7 cells) as the ubiquitous control genes respectively ( $\triangle \mathrm{CT}$ value; $\mathrm{n}=10$ Mean \pm SEM). The fold change for each target genes threshold cycle $(\Delta \mathrm{Ct})$ value followed by the normalization to the baseline control to measure the overall response as previously described [20].
Luminex studies: hMSC supernatants or PBMC supernatants were evaluated for cytokines and chemokines using Luminex multi-bead array technology ( $\& \& D$ Systems, Minneapolis, MN) as previously described [21,22], using a multi-array purchased which focused on IL-8, IL-6, macrophage inflammatory protein $1 \mathrm{a}(\mathrm{MIP}-1 \alpha)$, CCL-2, and stem cell factor 1 (SCF-1). Briefly, supernatants from samples were interfaced with luminescence beads with ratios of proprietary fluorophores. Each bead is specific for a cytokine or chemokine. As the sample interacts with the beads, the capture antibodies conjugated to the beads binds to the cytokine/chemokine which is followed by a second antibodies labeled with Phycoerythrin (PE). The detection of the cytokine/chemokine is based upon detection of the unique bead idea and the detection of the PA using a dual laser system with a lower end sensitivity of $3.2 \mathrm{pg} / \mathrm{ml}$. Each cytokine/chemokine is compared to its specific standard curve, also done in multiplex. Data is expressed as mean ( $\mathrm{pg} / \mathrm{ml} \pm \mathrm{SEM}, \mathrm{n}=10)$.

Aushon bioscience ciraplex studies: The Peripheral Blood Mononuclear Cells (PBMC) studies were evaluated using Aushon Bioscience Ciraplex ${ }^{\circ}$ Technology in which capture antibodies were spotted as a solid array chip, with the read-out being exquisitely sensitive chemi-luminescence. The advantage of this system was the small volume of sample required, the lower end sensitivity and the ease of the assay system. The sensitivity of the study was $1.0 \mathrm{pg} / \mathrm{ml}, \mathrm{n}=10$; MSC preparations and $\mathrm{n}=4$ PBMC preparations (see below). For the PBMC gene expression data, cells were cultured in vitro with and without LPS and hMSC supernatants. After 24 hours, cells were removed and supernatants were evaluated for cytokine and chemokines using the Aushon Ciraplex ${ }^{\bullet}$ multi-array technology using Trizol $^{\circledR}$ RNA extraction and TaqMan ${ }^{\circledR}$ real-time PCR assays.

Chemotaxis: PBMCs at $1 \times 10^{6}$ cells $/ \mathrm{ml}$ were put into the top chamber of a $0.4 \mu \mathrm{l}$ transwell tissue culture plate. In the bottom well, $0.5 \mathrm{mls}$ of fresh hMSC supernatant utilized as previously described 20. After 24 hours, the PBMCs in the top and the cells recruited into the bottom were counted for total cell count and made into cytospins to determine the cell differential. Cytospins were dried followed by staining with Wright Giemsa, which stains lymphocytes, monocytes, neutrophils, eosinophils and basophiles with a specific pattern.

PPAR $\gamma$ studies: The in vitro assay for measuring the overall impact of hMSCs on PPAR $\gamma$ was done using RAW-267 cells, a macrophage cell line with known parameters for expressing PPAR $\gamma$. Gene expression was done using real-time PCR as previously described [23].

Data evaluation and statistics: $\Delta \mathrm{CT}$ s were calculated by subtracting the relative cycling number of the probed sample (i.e. samples probed with IL-8, IL-6, CCL20 and CCL2) from the cycling number of the same sample probed with the background or housekeeping gene PPIA. In each group there were 10 different donor MSC preparations. $\Delta \Delta$ CTs were calculated by subtracting the $\Delta \mathrm{CT}$ of stimulated samples (i.e. LPS, I-172, I-172 LPS) from the unstimulated samples $\Delta$ CT for each preparation. Statistics were performed using GraphPad Prism 3.0 technology using non-parametric ANOVA analysis and standard T-cells when appropriate. Data is expressed as a Mean \pm Standard Error of the Mean (SEM) through non-parametric Mann-Whitney tests. $\mathrm{P}<0.05$ denotes significance.

\section{Results}

\section{In vivo potency and efficacy}

We have studied hMSC therapeutic potential in the infection/ inflammation murine model of inflammation and infection focusing on chronic Pseudomonas aeruginosa and Staphylococcus aureus 
pneumonia in WT and CF mice. Animals were infected followed 24 hours later by $10^{6} \mathrm{hMSCs}$ infused through the retro-orbital sinus and then monitored for 10 days prior to euthanasia for Bronchoalveolar Lavage (BAL). The CF BAL fluid had significantly more Colony Forming Units (CFUs) that the WT BAL fluid regardless of whether it was a gram negative (Pseudomonas aeruginosa) or gram positive (Staphylococcus aureus) infection ( $\mathrm{p} \leq 0.05, \mathrm{n}=4$ experiments, 10 mice per condition) [3]. When evaluating the inflammatory infiltrate of each of the models, both Pseudomonas aeruginosa and Staphylococcus aureus infections increased the total cell counts (Figure $1 \mathrm{~A}$ and $1 \mathrm{~B}$ respectively, which was decreased with hMSC treatment $(\mathrm{p} \leq 0.05, \mathrm{n}=4$ experiments, 10 mice per condition). When looking at the phenotype of the cells in the infection models, as previously described neutrophil recruitment was significantly higher in the $\mathrm{CF}$ model as compared to the WT controls (Figure $1 \mathrm{~A}$ and $1 \mathrm{~B}, \mathrm{p}<0.05$ for each, $\mathrm{n}=4$ experiments, 10 mice per condition). Treatment of each of the infection modeling systems with hMSCs resulted in a shift in the BAL differentials changing the lung milieu to preferentially attract macrophages (Figure $1 \mathrm{C} \mathrm{p} \leq$ $0.05, \mathrm{n}=4$ experiments, 10 mice per condition)) while decreasing the number of neutrophils (Figure 1D, $\mathrm{p} \leq 0.05, \mathrm{n}=4$ experiments, 10 mice per condition). Neutrophils are known to participate in the pathophysiologic process associated with CF lung disease. Macrophages are known to be important in infection resolution and attenuation of the inflammatory response.

\section{hMSCs are active at promoting cellular recruitment of a} specific phenotype

hMSCs enhance cellular recruitment overall, which could ultimately be beneficial or detrimental, depending on the cell type. In these studies, we monitored the ability of the hMSCs to recruit cells from peripheral blood mononuclear cells obtained from healthy volunteers using transwell plates of $0.45 \mu \mathrm{M}$. The hMSC supernatants significantly recruited cells from the upper chamber (Figure $2 \mathrm{~A}, \mathrm{p} \leq 0.05, \mathrm{n}=4$ ). Lymphocytes were most heavily recruited (Figure 2B, F variance of $\mathrm{p}=0.05$ ), followed by monocytes and lastly neutrophils. The preferential recruitment of lymphocytes may aide in lowering neutrophil elastase, translating to less lung damage.

\section{hMSC soluble mediators}

To determine how the hMSCs are contributing to the specific recruitment of cells, hMSCs were evaluated for cytokine secretion. The same supernatants that were utilized in the chemotaxis studies were evaluated in Luminex multiplex assays to determine their chemokine/ cytokine profile (Figure 3). The MSCs secreted a variety of cytokines/ chemokines that define the potential of the cells to contribute to their environment including IFN $\gamma$, IL-1 $\alpha$, IL-1 $\beta$, IL-2, IL-6, Il-8, IL-10, Il$12 \mathrm{p} 70, \mathrm{TNF} \alpha$ where we all significantly elevated relative to the control medium ( $\mathrm{p} \leq 0.05$ for all $\mathrm{n}=4$ ). The cytokines that were generated in the

\section{A: Pseudomonas aeruginosa}

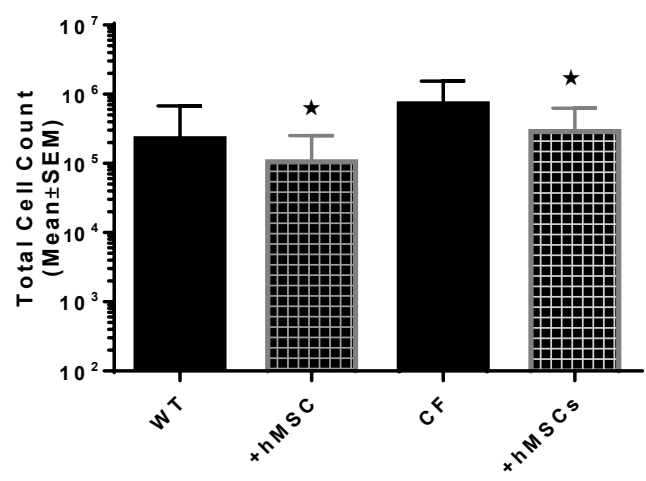

C : Relative \% Alveolar Macrophages

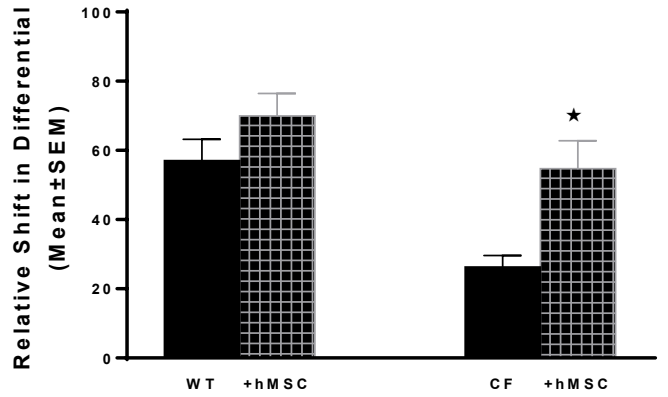

B: Staphylococcus aureus

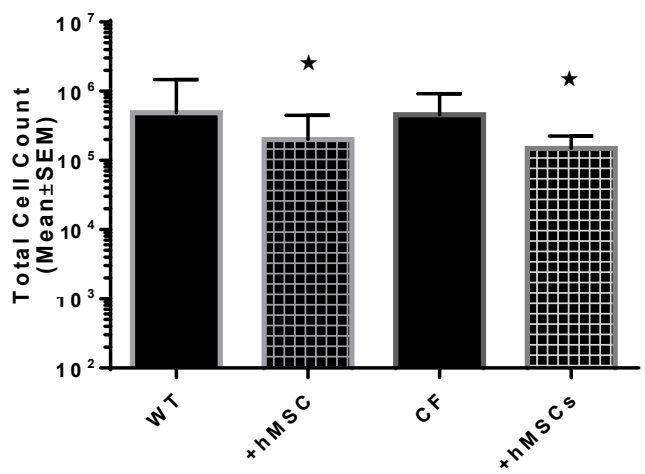

D : Relative \% PMNs

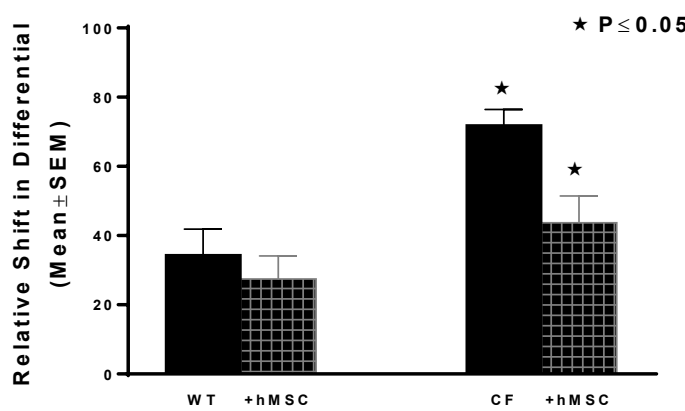

Figure 1: In Vivo Potency and Efficacy: Cftrtm1Kth (CF) and C57BL/6 (WT) mice were infected with either $10^{6}$. CFUs of Pseudomonas aeruginosa (A) or Staphylococcus aureus (B) and monitored for 10 days. At day 10 the total cell count (1A and $1 \mathrm{~B})$ were determined by bronchoalveolar lavage (BAL) using cytospin and H\&E staining from each of the different models. These studies were done 4 times with $n=10$ animals in each group using 4 different hMSC donors. Figure 1C and 1D focus on the differentials specific for the Pseudomonas aeruginosa model since it is prototypic of CF lung infection and inflammation. These data demonstrate that part of the impact of hMSCs is on re-directing the inflammatory infiltrate away from neutrophils toward having more macrophages. ( $\star: P \leq 0.05$, compared to the non-hMSC treated infected mice). 
A: Cells Recruited in Response to hMSCs

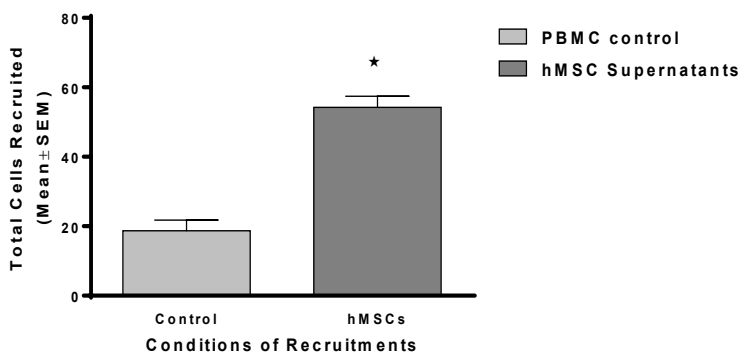

B: hMSC Specific Differential Recruitment

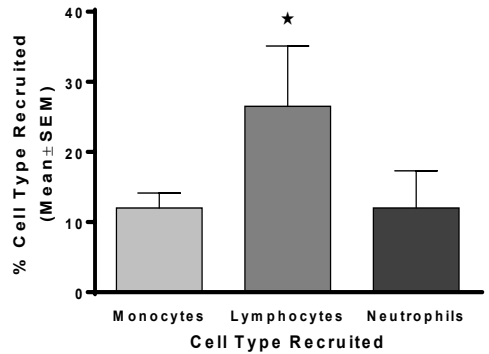

Figure 2: hMSCs and Cell Recruitment Studies. hMSCs supernatants $(n=4)$ were cultured in a transwell with peripheral blood monocytes from healthy volunteers. After 24 hours the cells were counted in both the bottom and top chambers to monitor the cell recruitment process. Data is the mean number of cells recruited in response to the MSC supernatants. ( $\star$ : $P \leq 0.05$, compared to the non-hMSC treated infected mice).

\section{Baseline MSC Supernatants Contain A Vareity of Cytokines}

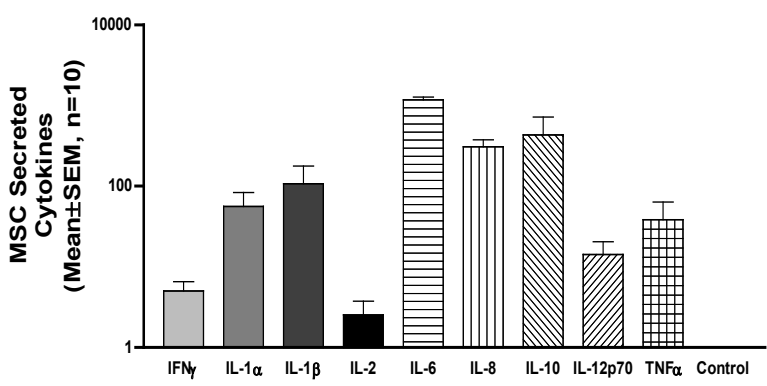

Figure 3: hMSC Cytokine Array. The same hMSC supernatants that were used for the chemotaxis studies were evaluated in a Luminex multiplex assay. Data are Mean \pm SEM $\mathrm{pg} / \mathrm{ml}$, for $\mathrm{n}=4$ different MSC donors. ( $\star$ : $\mathrm{P} \leq 0.05$, for all comparison with the medium control used to grow the hMSCs).

largest amount even without stimulation were IL-6 and IL-8 followed by IL- $1 \beta$ and IL-10 which were significantly higher than the medium control that is used to grow the hMSCs. The next series of studies focus on the expression of the chemokines that might predict how the hMSCs contribute to their environment and how the chemokine expression profile of the hMSCs may be impacted by the functionality of CFTR.

\section{hMSC IL-8 production and impact of CFTR}

IL-8 induces chemotaxis in target cells, shifting toward acute neutrophil influx at the sie of infection [24]. IL-8 has been shown to be significanly expressed in the absence of CFTR activity in a variety of other cells including epithelial cells [25]. hMSCs also secrete excessive levels of IL-8 when CFTR is inhibited as compared to control CFTR sufficient hMSCs (Figure 4 A, $-6.58 \pm 2.57$ versus $6.21 \pm 0.29$ respectively; $p \leq 0.05$, $\mathrm{n}=10$ ). Stimulation with LPS causes an increase in IL-8 expression in comparison to control mRNA, with CFTR inhibition causing a further increase (LPS : $0.29 \pm 1.35$ and LPS I-172: $0.40 \pm-1.31 ; p \leq 0.05$ for each; $\mathrm{n}=10$ ). Similar findings were determined through Luminex technology on secreted products, with differences between I-172 and LPS with I-172 also showing statistical significance (Figure $4 \mathrm{~B} p \leq 0.05$, $\mathrm{n}=10$ ).

\section{hMSC IL-6 production and impact of CFTR}

IL-6 is an acute phase protein, meaning that it induces other chemokines, such as IL-10. IL-6 has extensive anti-inflammatory functions, many of which could be beneficial in scenarios of chronic infection and inflammation such as CF [26]. IL-6 is downregulated in CFTR deficient cells in comparison to unstimulated mRNA, a difference of 1.04 cycles in ABI Taqman (Figure 5A, $\mathrm{p} \leq 0.05, \mathrm{n}=10$ ). Similarily, in comparing LPS stimualted hMSC mRNA, CFTR deficient cells expressed significantly less IL- 6 as opposed to LPS stimulated mRNA ( 1.84 cycles difference; $\mathrm{p} \leq 0.05, \mathrm{n}=10$ ). Similar results were obtained in secreted product data, with IL-6 downregulated in I-172 and LPS I-172 conditions in comparison to unstimulated controls (Figure 5B, $11225 \pm$ $13,11834 \pm 25$ and $12705 \pm 12.893$ respectively; $\mathrm{p} \leq 0.05, \mathrm{n}=10$ ).
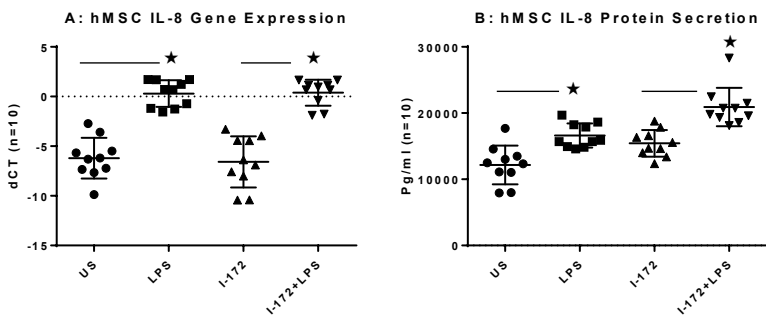

Figure 4: hMSCs IL-8 response defined by gene expression, secretion with and without CFTR activity and LPS exposure. hMSCs $(n=10)$ were cultured in the presence and absence of the CFTR inhibitor I-172 for 48 hours followed by stimulation with LPS. Cells were harvested and processed for gene expression $(\mathrm{dCT}, \mathrm{n}=10)$ and supernatants were evaluated for $\mathrm{IL}-8$ protein $(\mathrm{pg} / \mathrm{ml}, \mathrm{n}=10$. ( $\star$ : $\mathrm{P} \leq 0.05$, compared to the non-LPS or non-I-172 treated controls respectively. No statistical difference was observed between hMSCs with and without I-172 treated for baseline IL-8 gene expression of secretion).
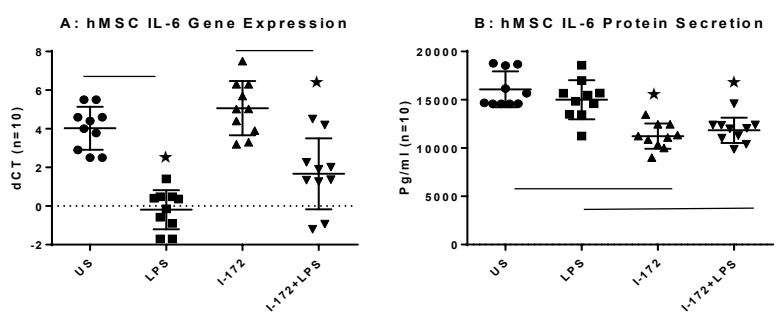

Figure 5: hMSCs IL-6 response defined by gene expression, secretion with and without CFTR activity and LPS exposure. hMSCs $(n=10)$ were cultured in the presence and absence of the CFTR inhibitor I-172 for 48 hours followed by stimulation with LPS. Cells were harvested and processed for gene expression $(\mathrm{dCT}, \mathrm{n}=10)$ and supernatants were evaluated for IL-6 protein $(\mathrm{pg} / \mathrm{ml}, \mathrm{n}=10)$. ( $\star$ : $\mathrm{P} \leq 0.05$, compared to the non-LPS or non-I-172 treated controls respectively. No statistical difference was observed between hMSCs with and without I-172 treated for baseline IL-6 gene expression, however hMSCs without functional CFTR secreted significantly lower levels of IL-6 protein, $P \leq 0.05$ ). 


\section{hMSCs CCL2 gene expression and secretion and the impact of CFTR activity}

CCL2 is a chemokine involved in the recruitment of monocytes and helper-T-cells, both of which are beneficial in scenarios of chronic inflammation [27]. CCL2 expression was significantly lowered with the addition of LPS, both in the case of otherwise unstimulated cells and in cells with I-172 inhibitor (Figure $6 \mathrm{~A},-0.03 \pm 1.0$ and $0.73 \pm 1.5$ respectively as compared to US: $4.49 \pm 0.98$ ( $\mathrm{p} \leq 0.05, \mathrm{n}=10)$. CCL2 expression was significantly lowered with the addition of LPS, both in the case of otherwise unstimulated supernatant and in supernatant with I-172 inhibitor ( $5402 \pm 22$., $5480 \pm 15$. respectively as compared to US: $6683 \pm 27, \mathrm{p} \leq 0.05, \mathrm{n}=10)$. Though not statistically significant, I-172 stimulation of hMSCs alone decreased CCL2 expression in Luminex (Figure 6B, $6337 \pm 14$ versus US: $6683 \pm 27 ; \mathrm{p} \leq 0.05, \mathrm{n}=10$ ).

\section{hMSCs CCL20 (MIP-3a) gene expression and secretion and the impact of CFTR activity}

CCL20 is chemotactic for lymphocytes and dendritic cells, while weakly recruiting neutrophils. CCL20 also has antimicrobial properties that would be beneficial in scenarios of acute infection. CCL20 is dependent on activation of amplified IL-6. There was a large decrease in expression between LPS stimulated cells and control cells, both with and without I-172 stimulations (Figure 7A, $9.9 \pm 1.7,4.9 \pm 0.98,5.2 \pm$ 1.3 respectively; $\mathrm{p} \leq 0.05, \mathrm{n}=10$ ). There was no significant difference in CCL20 expression in hMSCs between unstimulated and I-172 cells ( $\mathrm{p} \leq 0.05, \mathrm{n}=10)$. Similar data was determined in secreted products, with LPS stimulation providing significant decreases in expression of CCL20 chemokine as compared to control supernatant (Figure 7B, $\mathrm{p}$ $\leq 0.05 ; \mathrm{n}=10$ ).

\section{hMSCs impact on LPS stimulated PBMC cytokine profile}

PBMCs stimulated with LPS produced significant levels of IFN $\gamma$, IL$1 \alpha$, IL-1 $\beta$, IL-2, IL-6, IL-8, IL-10 IL-12p70 and TNFa ( $\mathrm{p} \leq 0.05$ for all) relative to control unstimulated PBMCs (data not shown). Proinflammatory and lymphocyte activating cytokines IL-1 $\alpha$, IL-1 $\beta$, IL-2 IL-6, TNF- $\alpha$, IFN- $\gamma$ were decreased when the PBMCs were cultured in the presence of hMSCs (Figure 8). Cytokine TNFa was decreased from $216 . \pm 4.3$ to $140 \pm 3.4 \mathrm{pg} / \mathrm{ML}$ with hMSC stimulation post LPS stimulation. Cytokine INF $\gamma$ decreased with hMSC stimulation post LPS stimulation from $98 \pm 2$ to $83 \pm 3.3$. Cytokines were minimal in the non -bacterial stimulated samples (no LPS), with levels similarly minimal in both unstimulated hMSC and control conditions. hMSCs down-regulate the inflammatory response of monocytes exposed to bacterial toxin such as LPS which is summarized in Table 1.
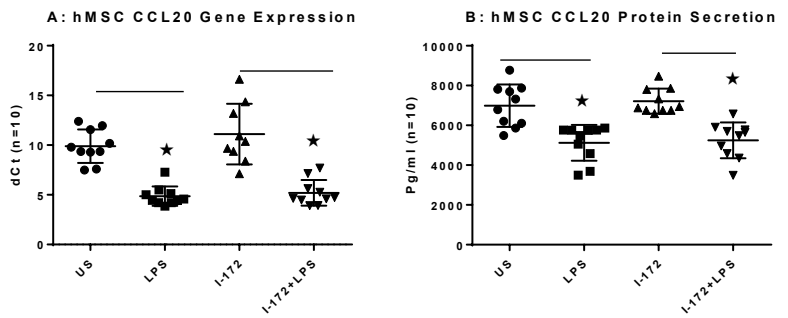

Figure 6: hMSCs CCL20 response defined by gene expression, secretion with and without CFTR activity and LPS exposure. hMSCs $(n=10)$ were cultured in the presence and absence of the CFTR inhibitor I-172 for 48 hours followed by stimulation with LPS. Cells were harvested and processed for gene expression and supernatants were evaluated for CCL20 protein. ( $\star$ : $P \leq 0.05$, compared to the non-LPS or non-I-172 treated controls respectively. No statistical difference was observed between hMSCs with and without I-172 treated for baseline CCL20 gene expression of secretion).
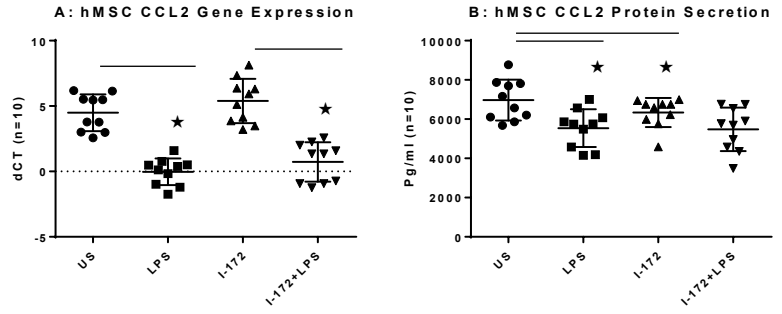

Figure 7: hMSCs CCL2 response defined by gene expression, secretion with and without CFTR activity and LPS exposure. hMSCs $(n=10)$ were cultured in the presence and absence of the CFTR inhibitor I-172 for 48 hours followed by stimulation with LPS. Cells were harvested and processed for gene expression $(\mathrm{dCT}, \mathrm{n}=10)$ and supernatants were evaluated for CCL2 protein $(\mathrm{pg} / \mathrm{ml}$, $n=10$ ). ( $\star$ : $P \leq 0.05$, compared to the non-LPS or non-I-172 treated controls respectively. No statistical difference was observed between hMSCs with and without I-172 treated for baseline CCL2 gene expression, however again blocking CFTR activity decreased overall secretion, $\mathrm{P} \leq 0.05$ ).

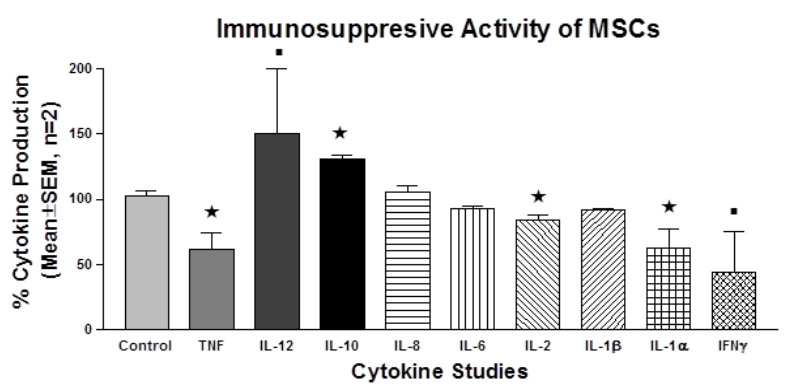

Figure 8: PBMCs and hMSCs- Suppression of Response to LPS Exposure Ex vivo. Peripheral blood mononuclear cells were obtained from healthy volunteers $(n=4)$ and cultured with and without hMSCs after being stimulated for 24 hours with LPS. Data is the change in cytokine expression post-hMSC treatment as compared to PBMCs without hMSCs. ( $\star$ : P $\leq 0.05$, compared to the control PBMCs without hMSC treatment, $\star: P \leq 0.06$ compared to the control PBMCs without hMSC treatment. IL-1 $\beta$, IL- 6 and IL-8 were not different from the control.

\begin{tabular}{|cc|c|}
\hline Cytokine & \multicolumn{2}{c|}{ Concentration Change } \\
\hline \multicolumn{3}{|c|}{ Pro- Inflammatory } \\
\hline IL-1alpha & $-11.50 \%$ \\
\hline IL-1Beta & $-8.25 \%$ \\
\hline IL-6 & $-6.85 \%$ \\
\hline TNF-alpha & $-39.20 \%$ \\
\hline \multicolumn{3}{|c|}{ TH1 Activators } \\
\hline IFN-2 & \\
\hline \multicolumn{3}{|c|}{ Anti-Inflammatory } \\
\hline
\end{tabular}

Table 1: Immune Redirection by hMSCs.

The absence of CFTR decreases PPAR $\gamma$ gene expression which can be repaired by hMSCs

PPAR $\gamma$ is constitutively expressed in alveolar macrophages to attenuate the immune response to constant bombardment by environmental antigens $[28,29]$. In scenarios of chronic inflammation, PPAR $\gamma$ becomes deficient [30]. PPAR $\gamma$ has been shown to be deficient in macrophages and epithelial cells [31-35]. We generated a model of this observation using the CFTR blocker I-172, which decreased PPAR $\gamma$ in WT macrophages (Figure 9A). When CFTR activity is blocked PPAR $\gamma$ 
A Inhibition of CFTR Decreases PPAR $\gamma$ Gene Expression

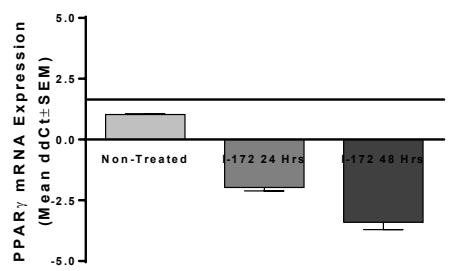

hMSC Supernatants Induce PPARy and Turn Off TNFa in Macrophages

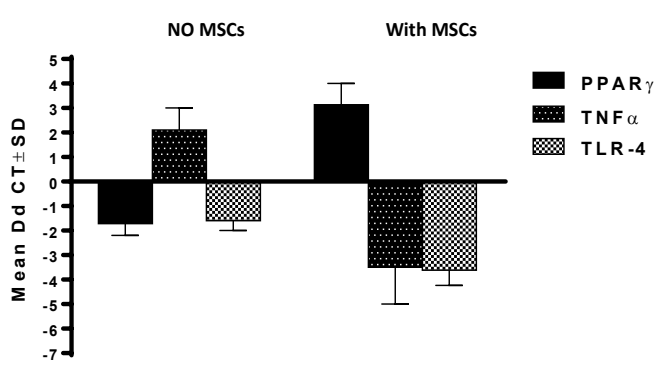

Figure 9: CFTR Activity and Expression Impact PPARy Expression which can be reversed by hMSCs Therapeutics. Bone marrow derived macrophages from CF and WT mice were processed for CDNA and monitored for PPARY gene expression as compared to GAPDH $(A, n=4)$. Bone marrow derived macrophages treated with the CFTR inhibitor I-172 become deficient in PPARY by 24 hours $(p<0.05)$ which suggest CFTR function is associated with PPARY expression $(A, n=4)$. The deficiency in PPARy correlates with increased TNFa $(\star P \leq 0.05)$ hMSCs cultured in scenarios of deficient PPARY express increased TNFa, but less TLR-4. The deficient PPARY can be rescued with hMSCs (B; $\star \mathrm{P} \leq 0.05, \mathrm{~B}, \mathrm{n}=4)$ contributing to less TNFa. There was also a trend towards decreased TLR-4 with the hMSCs $(\star p=0.06, n=4)$.

\section{Anti-Microbial and Anti-Inflammatory Potency of hMSCs}

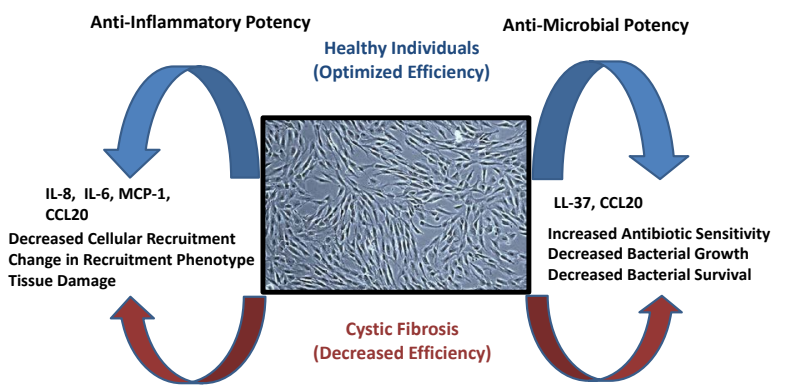

Figure 10: Theoretical Schematic of The Syngergistic Potential of hMSC Down Regulating Inflammation and Managing Infection. Infection with or without inflammation in CF is poorly regulated and contributes to disease pathogenesis. hMSCs produce soluble mediators which redefine the tissue niche, enhancing macrophage recruitment which may can participate in host immunity. In CF, hMSCs have altered levels of soluble mediators relative to the control suggesting that the immune function of hMSCs in CF maybe alters again re-re-enforcing the notion of allogeneic therapeutics in the context of genetic diseases.

levels decrease as shown in the comparison of treated (I-172 inhibited) versus non-treated mRNA PPAR $\gamma$ expression at 24 and 48 hours post treatment with inhibitor. In Figure 9, the comparison of the non- treated ddCT value of $0.925 \pm 0.3,24$ hours and 48-hour treatment to the treated groups, there was significantly less PPAR $\gamma(-2.12 \pm-0.3$ and $-3.52 \pm 0.2$ respectively for 24 and 48 hours, $p \leq 0.05)$. We have further established that hMSCs have the capacity to rescue deficient PPAR $y$ expression whether the deficient is due to endotoxin induced down-regulation (Figure 9B) or deficient CFTR activity. Bone marrow derive macrophages were treated with LPS which resulted in decreased PPAR $\gamma$, TLR-4 expression while TNFa expression is increased as predicted. When hMSCs are added, PPAR $\gamma$ expression was increased while turning down the expression of both TNFa and TLR-4, decreasing the pro-inflammatory phenotype of the macrophages. The increase in TNFa specifically correlated with the decrease in PPAR $\gamma$ ddCT value (normalized to initial start time CT value) from 6 to 24 hours $(-1.87 \pm$ 0.74 and $3.35 \pm 0.46$ respectively; Mean \pm SEM, $\mathrm{p} \leq 0.05$ ).

\section{Discussion}

We and others have previously demonstrated that hMSCs secrete products that are anti-inflammatory and antimicrobial, making them a suitable candidate to treat diseases associated with severe chronic infection and inflammation such as CF. Defects in the CFTR gene in CF result in increased susceptibility to pulmonary infections from Pseudomonas aeruginosa, Staphylococcus aureus and Streptococcus pneumonia [24]. The inability to resolve these infections and the ensuing over-zealous inflammatory response is the major cause of morbidity and mortality in CF. Our previous data has shown that hMSCs decrease inflammation in the in vivo murine model of $\mathrm{CF}$ chronic Pseudomonas aeruginosa infection and inflammation. The studies outlined in this manuscript focus on understanding the diversity in the hMSCs anti-inflammatory effectiveness. We have previously shown that growth conditions impact the overall antimicrobial effectiveness of hMSCs and that deficient CFTR function can also decrease antimicrobial potency [24]. Furthering these studies on hMSC phenotype, we have demonstrated that hMSCs are potent inflammatory mediators, and have contributed to defining the complexity of these cells. Our studies demonstrated that: 1) hMSCs have the capacity to alter the inflammatory response in both a gram negative (Pseudomonas aeruginosa) and gram positive (Staphylococcus aureus) murine modeling system of CF; 2) hMSCs have the capacity to alter their environment by directly recruiting cells of a specific type recruiting more lymphocytes than monocytes or neutrophils; 3) hMSCs produce a wide variety of factors that have the capacity to define the host response; 4) hMSCs are anti-inflammatory, producing anti-inflammatory chemokines, increasing IL-6 and CCL2 expression, while decreasing IL-8 expression; 5) hMSCs also produce the chemokine CCL20 (MIP- $\alpha$ ) which has not only chemotactic properties but also has been shown to be antimicrobial; 6) Deficient CFTR activity alters the expression of all of these cytokines as well as the capacity of the hMSCs to respond to LPS and gram negative bacteria; 7). Deficient CFTR results in the deficiency of the anti-inflammatory transcriptional regulator PPAR $\gamma$ contributing to inflammation by producing factors such as TNFa; 8) h MSCs have the capacity to rescue PPAR $\gamma$ expression as a potential mechanism associated with the MSC anti-inflammatory effectiveness in CF and other chronic inflammatory diseases.

Previous studies have implicated the anti-inflammatory and antimicrobial activity of the hMSCs in both animal models and in preliminary clinical trials [36-38]. In our own studies, we have been able to demonstrate that not only do hMSCs impact inflammation and infection they also alter the direction of inflammatory cell recruitment. In WT and CF mice infected with Pseudomonas aeruginosa, hMSCs decreased both bacterial burden and inflammation [14]. Mice given 
the hMSCs had significantly less bacterial burden than mice treated with saline along with attenuated inflammation. In the studies presented in this manuscript, we have demonstrated that hMSCs are active contributors to the host environment by producing cytokines and chemokines that participate, in the host response to pathogen exposure. We believe that the contribution of the hMSCs will be disease specific since in our hands using the same hMSC preparations we have been able to identify very different end-points of hMSC effectiveness whether it is in the context of infection (as in the CF studies) or in acute inflammation 12 or chronic asthma [38]. Certainly our studies demonstrate the unique capacity of the hMSCs to alter inflammation regardless of the inciting source of the pathophysiology.

There are several aspects that need to be considered when thinking about hMSC-based therapeutics including how the cells are cultured and administered [36,39] but also important to consider the source of the hMSC preparation. Several reviews have outlined the unique attributes of MSCs and their specific source and processing and the outcome on clinical potency and efficacy $[37,40]$. In our studies, we have demonstrated previously that hMSCs deficient in CFTR activity have decreased anti-microbial potency and efficacy 3 which may have important implications into whether the autologous therapeutics can be utilized for CF hMSC clinical trials. This may also relate to other diseases in which there is a genetic association with the pathogenicity and may be the reasoning behind the overall variability in hMSC clinical efficacy in clinical trials. The results outlined in this manuscript explore the issue of CFTR expression on hMSC anti-inflammatory potency. Our data indicate that CF hMSCs do not behave similarly to healthy hMSCs in response to bacterial stimulation with healthy and CF hMSCs express different amounts of IL-6, CCL2, CCL20 and IL-8 $[41,42]$. With bacterial stimulation, CF cells express a greater amount of IL-8 than healthy hMSCs when both are exposed to bacteria. The CF hMSCs express less IL-6, CCL2 and CCL20 than healthy hMSCs with bacterial stimulation. Although CF hMSCs and healthy hMSCs express different amounts of IL-6 CCL2, and IL-8, both types of MSCs express roughly equal amounts of CCL20 which may be important in the chemotactic and antimicrobial functions of these chemokine/cytokines $[27,43]$. The production of chemokines and cytokine mediators will have a contributory role in defining the tissue niche as a response to the inflammatory stimulus. The contributory fingerprints of the hMSCs secreted products will ultimate change how the host will manage the inflammatory process.

The difference in expression of cytokines between healthy and CF hMSCs in response to bacterial stimulation indicates that autologous hMSCs are unlikely to be a successful form of therapy for CF patients. The data also suggests the impact of CFTR deficient activity throughout CF patient's body, not only at the level of the immune system but also the functionality of the autologous hMSCs. This implies an additional contribution of deficient CFTR on CF pathophysiology, encompassing not only the primary major consequence of the defect on the epithelium, but also the additive impact on the CF immune system and the multipotent cell source of hMSCs

In the pursuit of the mechanistic action of the hMSCs as an antiinflammatory adjuvant therapeutic, we have begun to identify the potential of hMSCs to alter PPAR $\gamma$ expression in macrophages which is an important regulator of inflammation in chronic inflammation [44]. In the lung, PPAR $\gamma$ is constitutively expressed in alveolar macrophages in order to attenuate the host response to the continuous exposure to environmental insult $[23,33]$. PPAR $\gamma$ quiets inflammation through transcriptional repression of NFKB preventing the ability of $\mathrm{NF \kappa B}$ to initiating transcription from its promoter regions which are involved with pro-inflammatory gene expression [45]. In scenarios of chronic inflammation, PPAR $\gamma$ becomes deficient in macrophages which contributes to the uncontrolled expression of pro-inflammatory genes by NFkB [35], a pathophysiological mechanism described in a variety of chronic inflammatory diseases $[46,47]$. Like our studies it has been shown that CF macrophages are deficient in PPAR $\gamma$ [35]. We have been able to demonstrated that hMSCs can up-regulate the expression of PPAR $\gamma$, in macrophages which in turn has the capacity to down-regulate inflammation regardless of the routes associated with the down-regulation of this inflammation rheostat. Theoretically, the hMSCs likely produce soluble mediators which alter the macrophage phenotype, enhancing the PPAR $\gamma$ expression, allowing for efficient modification of pro-inflammatory cytokine production through theprevention of NFkB promoter activation. In either case of LPS stimulated mouse bone marrow derived macrophages or human peripheral blood mononuclear cells, hMSCs decreased proinflammatory cytokine production. The direct mechanisms of these effects are the focus of on-going work in our laboratory.

hMSCs have anti-inflammatory potential which has been documented in several different in vivo and in vitro modeling systems [48]. We propose that one of the mechanisms associated with this hMSC function is the capacity to up-regulate the expression of PPAR $\gamma$. How CFTR might impact this effect of hMSCs will be pursued in the context of our investigator initiated clinical trial of hMSCs in CF. Further, these studies are consistent with previous benefits of hMSCs in scenarios of Acute Lung Injury (ALI) which is a disease of significant morbidity and mortality in intensive care patients [37]. ALI is defined by the acute onset of pulmonary infiltrates with severe hypoxemia. In a mouse model of ALI, Lipopolysaccharide (LPS) derived from the bacterium Escherichia coli endotoxin was put into the lung followed by administration of murine hMSCs several hours later. Mice treated with murine MSCs had decreased inflammation $[49,50]$. The pro-inflammatory response to the LPS endotoxin was decreased, while the resolution response and anti-inflammatory cytokines were enhanced [18]. These studies suggest the innovative use of adult human MSCs in the treatment of lung diseases associated with infection and inflammation.

\section{Conclusions}

hMSCs are active in their environment secreting products that define the host milieu and capacity to regulate immunity and cellular response. The capacity to down-regulate inflammation is reproducible regardless of the modeling system using mouse bone marrow derived macrophages or human peripheral blood mononuclear cells going across species with hMSCs. The capacity of the hMSCs to secrete cytokines and respond to pathogen endotoxin like LPS is impacted by CFTR activity, suggesting that CF hMSCs may not be appropriate for autologous therapy. The inefficiency of the CF hMSCs in these systems along with the established antimicrobial activity, implies that innate hMSC function may altered in CF, potentially contributing to disease. hMSCs are environmentally sensitive and environmentally pro-active emphasizing the complexity of their function and potential therapeutic impact. Future studies will pursue the impact of CFTR on hMSC activity and how hMSC regulates the combined anti-inflammatory and antimicrobial milieu in vivo.

\section{Acknowledgements}

We appreciate the expertise and the services provided by the CTSC Bioanalyte Core and the CF Animal Core. The funding for this research was graciously provided by the David and Virginia Baldwin Fund, The National Center for Stem Cell and Regenerative Medicine, The Case 
Citation: Sutton MT, Fletcher D, Episalla N, Auster L, Folz M, et al. (2017) Mesenchymal Stem Cell Soluble Mediators and Cystic Fibrosis. J Stem Cell Res Ther 7: 400. doi: 10.4172/2157-7633.1000400

Western Reserve Vision Fund, Rainbow Babies and Children's Hospital Summer Programs in Undergraduate Research, Rainbow Babies and Children's Hospitals Strategic Innovator Award and National Institutes of Health HL: R21:104362.

\section{Author Contributions}

Morgan T. Sutton: Data Generation, Conception and Design, Collection and Assembly of Data, Data Analysis and Interpretation, Manuscript Writing

David Fletcher: Data Generation Collection and/or Assembly of Data, Data Analysis and Interpretation. Nicole Episalla: Data Generation, Collection and Assembly of Data.

Lauren Auster: Provision of Study Materials, Manuscript Review.

Michael Folz: Manuscript Review Data Analysis and Interpretation.

Varun Roy: Generation of Peripheral Blood Mononuclear Studies, Data Analysis and Interpretation. Rolf vanHeeckeren: Provision of Study Materials, Collection and/or Assembly of Data

Donald Lennon: Provision of Study Materials

Arnold Caplan: Financial Support, Provision of Study Materials, Data Analysis and Interpretation, Final Approval of Manuscript.

Tracey L. Bonfield: Conception and Design, Financial Support, Administrative Support, Provision of Study Materials, Data Analysis and Interpretation, Manuscript Writing, Final Approval of Manuscript.

\section{Disclosure of Potential Conflicts of Interest}

The authors listed have no conflicts of interest to report.

\section{References}

1. Caplan Al (2009) Why are MSCs therapeutic? New data: new insight. J Patho 217: 318-324. [PubMed]

2. Tomic S, Djokic J, Vasilijic S, Vucevic D, Todorovic V, et al. (2011) Immunomodulatory properties of mesenchymal stem cells derived from dental pulp and dental follicle are susceptible to activation by toll-like receptor agonists. Stem Cells Dev 20: 695-708. [PubMed]

3. Sutton MT, Fletcher D, Ghosh SK, Weinberg A, van Heeckeren R, et al. (2016) Antimicrobial Properties of Mesenchymal Stem Cells: Therapeutic Potential for Cystic Fibrosis Infection, and Treatment. Stem Cells Int 2016: 5303048. [PubMed]

4. Nichols D, Chmiel J, Berger M (2008) Chronic inflammation in the cystic fibrosis lung: alterations in inter- and intracellular signaling. Clin RevAllergy Immunol 34: 146-162. [PubMed]

5. Drumm ML, Ziady AG, Davis PB (2012) Genetic variation and clinical heterogeneity in cystic fibrosis. Annu Rev Pathol 7: 267-282. [PubMed]

6. Dasenbrook EC, Checkley W, Merlo CA, Konstan MW, Lechtzin N, et al. (2010) Association between respiratory tract methicillin-resistant Staphylococcus aureus and survival in cystic fibrosis. JAMA 303: 2386-2392. [PubMed]

7. Goss $\mathrm{CH}$, Muhlebach MS (2011) Review: Staphylococcus aureus and MRSA in cystic fibrosis. JCystFibros 10: 298-306. [PubMed]

8. Kotha K, Clancy JP (2013) Ivacaftor treatment of cystic fibrosis patients with the G551D mutation: a review of the evidence. Ther Adv Respir Dis 7: 288-296. [PubMed]

9. Cosgrove S, Chotirmall SH, Greene CM, McElvaney NG (2011) Pulmonary proteases in the cystic fibrosis lung induce interleukin 8 expression from bronchial epithelial cells via a heme/meprin/epidermal growth factor receptor/ Toll-like receptor pathway. J Biol Chem 286: 7692-7704. [PubMed]

10. Freedman MS, Bar-Or A, Atkins HL, Karussis D, Frassoni F, et al. (2010) The therapeutic potential of mesenchymal stem cell transplantation as a treatment for multiple sclerosis: consensus report of the International MSCT Study Group. Mult Scler 16: 503-510. [PubMed]
11. Roddy GW, Oh JY, Lee RH, Bartosh TJ, Ylostalo J, et al. (2011) Action at distance: systemically administered adult stem/progenitor cells (MSCs) reduce inflammatory damage to the cornea without engraftment and primarily by secretion of TNF-alpha stimulated gene/protein 6. Stem Cells 29: 1572-1579. [PubMed]

12. Bonfield TL, Nolan Koloze MT, Lennon DP, Caplan Al (2010) Defining human mesenchymal stem cell efficacy in vivo. J Inflamm (Lond) 7: 51. [PubMed]

13. Bonfield TL, Caplan Al (2010) Adult mesenchymal stem cells: an innovative therapeutic for lung diseases. Discov Med 9: 337-345. [PubMed]

14. Bonfield TL, Ghosh SK, Weinberg A, Caplan Al (2013) Cell Based Therapy Aides in Infection and Inflammation Resolution in The Murine Model of Cystic Fibrosis Lung Disease. Stem Cell Discov.

15. Lennon DP, Caplan $\mathrm{Al}$ (2006) Isolation of human marrow-derived mesenchymal stem cells. Exp Hematol 34: 1604-1605. [PubMed]

16. Caimi PF, Reese J, Lee Z, Lazarus HM (2010) Emerging therapeutic approaches for multipotent mesenchymal stromal cells. Curr Opin Hematol 17 505-513. [PubMed]

17. Bonfield TL, Barna BP, John N, Malur A, Culver DA, et al Suppression of activin $A$ in autoimmune lung disease associated with anti-GM-CSF. J Autoimmun. 2006;26(1):37-41. [PubMed].

18. van Heeckeren AM, Schluchter MD (2002) Murine models of chronic Pseudomonas aeruginosa lung infection. Lab Anim 36: 291-312. [PubMed]

19. Bonfield TL, Thomassen MJ, Farver CF, Abraham S, Koloze MT, et al. (2008) Peroxisome proliferator-activated receptor-gamma regulates the expression of alveolar macrophage macrophage colony-stimulating factor. J Immunol 181 235-242. [PubMed]

20. Bonfield TL, John N, Malur A, Barna BP, Culver DA, et al. (2004) Elevated monocyte chemotactic proteins 1,2 , and 3 in pulmonary alveolar proteins are associated with chemokine receptor suppression. Clin Immunol 114: 79-85. [PubMed]

21. Bonfield TL, John N, Barna BP, Kavuru MS, Thomassen MJ, et al. (2005) Multiplexed particle-based anti-granulocyte macrophage colony stimulating factor (GM-CSF) assay: A pulmonary diagnostic test. Clin Diagn Lab Immuno 12: 821-824. [PubMed]

22. Rieder F, Nonevski I, Ma J, Ouyang Z, West G, et al. (2014) T-helper 2 cytokines, transforming growth factor $\beta 1$, and eosinophil products induce fibrogenesis and alter muscle motility in patients with eosinophilic esophagitis. Gastroenterology 146: 1266-1277. [PubMed]

23. Bonfield TL, Farver CF, Barna BP (2007) PPAR_g is deficient in alveolar macrophages from patients with alveolar proteinosis. Am J Respir Cell Mo Biol 29: 677-682.

24. Car BD, Meloni F, Luisetti M, Semenzato G, Gialdroni-Grassi G, et al. (1994) Elevated IL-8 and MCP-1 in the bronchoalveolar lavage fluid of patients with idiopathic pulmonary fibrosis and pulmonary sarcoidosis. AmJ Respir Crit Care Med 149: 655-659. [PubMed]

25. Saadane A, Eastman J, Berger M, Bonfield TL (2011) Parthenolide inhibits ERK and AP-1 which are dysregulated and contribute to excessive IL-8 expression and secretion in cystic fibrosis cells. J Inflamm (Lond) 8: 26. [PubMed]

26. Niemand C, Nimmesgern A, Haan S, Fischer P, Schaper F, et al. (2003) Activation of STAT3 by IL- 6 and IL-10 in primary human macrophages is differentially modulated by suppressor of cytokine signaling 3 . J Immunol 170 3263-3272. [PubMed]

27. Daly C, Rollins BJ (2003) Monocyte chemoattractant protein-1 (CCL2) in inflammatory disease and adaptive immunity: therapeutic opportunities and controversies. Microcirculation 10: 247-257. [PubMed]

28. Daynes RA, Jones DC (2002) Emerging roles of PPARs in inflammation and immunity. Nat Rev Immunol 2: 748-759. [PubMed]

29. Berger J, Moller DE (2002) The mechanisms of action of PPARs. Ann Rev Med 53: 409-435. [PubMed]

30. Zhang X, Young HA. PPAR and immune system--what do we know? Int Immuno pharmacol 2: 1029-1044. [PubMed]

31. Perez A, van Heeckeren AM, Nichols D, Gupta S, Eastman JF, et al. (2008) Peroxisome proliferator-activated receptor-\{gamma $\}$ in cystic fibrosis lung epithelium. Am J Physiol Lung Cell Mol Physiol 295: L303-L313. [PubMed] 
Citation: Sutton MT, Fletcher D, Episalla N, Auster L, Folz M, et al. (2017) Mesenchymal Stem Cell Soluble Mediators and Cystic Fibrosis. J Stem Cell Res Ther 7: 400. doi: 10.4172/2157-7633.1000400

Page 9 of 9

32. Malur A, Abraham S, Culver DA (2005) Interferon -gamma (IFNgamma) represses PPARgamma expression in sarcoidosis. Proc Am Thorac Soc 2: A843.

33. Culver DA, Barna BP, Raychaudhuri B, Bonfield TL, Abraham S, et al. (2004) Peroxisome proliferator-activated receptor gamma activity is deficient in alveolar macrophages in pulmonary sarcoidosis. Am J Respir Cell Mol Biol 30: 1-5. [PubMed]

34. Bonfield TL, Farver CF, Barna BP, Malur A, Abraham S, et al. (2003) Peroxisome proliferator-activated receptor-gamma is deficient in alveolar macrophages from patients with alveolar proteinosis. Am J Respir Cell Mol Biol 29: 677-682. [PubMed]

35. Andersson C, Zaman MM, Jones AB, Freedman SD (2008) Alterations in immune response and PPAR/LXR regulation in cystic fibrosis macrophages. $J$ Cyst Fibros 7: 68-78. [PubMed]

36. Kean TJ, Lin P, Caplan AI, Dennis JE (2013) MSCs: Delivery Routes and Engraftment, Cell-Targeting Strategies, and Immune Modulation. Stem Cells Int 2013: 732742.

37. Matthay MA, Thompson BT, Read EJ, McKenna DH Jr, Liu KD, et al. (2010) Therapeutic potential of mesenchymal stem cells for severe acute lung injury. Chest 138: 965-972. [PubMed]

38. Bonfield TL, Koloze M, Lennon DP, Zuchowski B, Yang SE, et al. (2010) Human mesenchymal stem cells suppress chronic airway inflammation in the murine ovalbumin asthma model. Am J Physiol Lung Cell Mol Physiol 299: L760-L770. [PubMed]

39. Zagoura DS, Trohatou O, Bitsika V, Makridakis M, Pappa KI, et al. (2013) AFMSCs fate can be regulated by culture conditions. Cell Death Dis 4: e571. [PubMed]

40. Caplan Al (2013) Adult mesenchymal stem cells and the NO pathways. Proc NatlAcad Sci USA 110: 2695-2696. [PubMed]
41. Moser B, Wolf M, Walz A, Loetscher $P$ (2004) Chemokines: multiple levels of leukocyte migration control. Trends Immunol 25: 75-84. [PubMed]

42. Zeng SL, Wang LH, Li P, Wang W, Yang J (2015) Mesenchymal stem cells abrogate experimental asthma by altering dendritic cell function 5 . Mol Med Rep 12: 2511-2520. [PubMed]

43. Vulcano M, Struyf S, Scapini $P$, Cassatella M, Bernasconi $S$, et al. Unique regulation of CCL18 production by maturing dendritic cells. J Immunol 170: 3843-3849. [PubMed]

44. Li M, Pascual G, Glass CK (2000) Peroxisome proliferator-activated receptor gamma-dependent repression of the inducible nitric oxide synthase gene. Mol Cell Biol20: 4699-4707. [PubMed]

45. Welch JS, Ricote M, Akiyama TE, Gonzalez FJ, Glass CK (2003) PPARgamma and PPARdelta negatively regulate specific subsets of lipopolysaccharide and IFN-gamma target genes in macrophages. Proc Natl Acad Sci USA 100: 67126717. [PubMed]

46. Belvisi MG, Hele DJ, Birrell MA (2006) Peroxisome proliferator-activated receptor gamma agonists as therapy for chronic airway inflammation. Eur J Pharmacol. 533: 101-109. [PubMed]

47. Clark RB (2002) The role of PPARs in inflammation and immunity. J Leukoc Biol 71: 388-400. [PubMed]

48. Dimarino AM, Caplan AI, Bonfield TL (2013) Mesenchymal Stem Cells in Tissue Repair. Front Immunol 4: 201. [PubMed]

49. Krasnodembskaya A, Song Y, Fang X, Gupta N, Serikov V, et al. Antibacteria effect of human mesenchymal stem cells is mediated in part from secretion of the antimicrobial peptide LL-37. Stem Cells 28: 2229-2238. [PubMed]

50. Matthay MA, Goolaerts A, Howard JP, Lee JW (2010) Mesenchymal stem cells for acute lung injury: preclinical evidence. Crit Care Med 38: S569-S573. [PubMed] 